\title{
Application of Generalized Measure of 'Useful' R-norm Inaccuracy and Total Ambiguity
}

\author{
Saima Manzoor \\ Department of Statistics, University of Kashmir, Srinagar, India \\ E-mail: saimam.stsc@gmail.com
}

\section{Safina Peerzada and Mirza A. K. Baig}

Department of Statistics, University of Kashmir, Srinagar, India

E-mail: \{sapezad, baigmak\}@gmail.com

Received: 25 March 2019; Accepted: 12 August 2019; Published: 08 February 2021

\begin{abstract}
In the present paper, we introduce generalized measure of 'useful' R-norm inaccuracy having two parameters and its analogue 'useful' R-norm total ambiguity measure by merging together the concepts of probability, fuzziness, Rnorm, 'useful' information and inaccuracy. Along with the basic properties, some other important properties of these two proposed measures are stated. These measures are generalizations of some well-known inaccuracy measures. Further, the monotonic behaviour of the proposed 'useful' R-norm inaccuracy measures is studied and the graphical overview is given. The measure of information improvement for both the measures is also obtained. Lastly, the application of 'useful' R-norm total ambiguity measure is presented in terms of multi-criteria decision making. For all the numerical calculations $\mathrm{R}$ software is used.
\end{abstract}

Index Terms: Fuzzy sets, inaccuracy measures, R-norm information measures, 'useful' information measures, total ambiguity measures, multi-criteria decision making.

\section{Introduction}

The concept of inaccuracy measure was first introduced by Kerridge [1] as an extension of Shannon's [2] measure of information. Kerridge [1] regarded inaccuracy as a quantity of measuring missing information. When the probabilities of the outcomes of a random experiment are stated by an experimenter, his statement may be imprecise in two ways. Firstly, his statement may be vague and secondly he may have some incorrect information. The suitable measure for dealing with these two kinds of errors is Kerridge's [1] inaccuracy measure which is given as

$$
I(P ; Q)=-\sum_{i=1}^{n} p_{i} \log q_{i}
$$

Here $P=\left(p_{1}, p_{2}, \ldots, p_{n}\right) \& Q=\left(q_{1}, q_{2}, \ldots, q_{n}\right)$ represent the true and asserted probability distributions associated with the events $Z=\left(Z_{1}, Z_{2}, \ldots, Z_{n}\right)$. Suppose the experimenter considers the importance of $Z_{i}$ events (irrespective of their true and asserted probability) and assigns a non-negative number $u_{i}(>0)$ to each $Z_{i} \cdot u_{i}$ represents the importance of $Z_{i}$. In this regard, Hooda [3], defined the following 'useful' inaccuracy measure.

$$
I(P ; Q)=-\frac{\sum_{i=1}^{n} u_{i} p_{i} \log q_{i}}{\sum_{i=1}^{n} u_{i} p_{i}} .
$$

In the context of fuzzy set theory, which was originally developed by Zadeh [4], inaccuracy measure is called total ambiguity measure. Corresponding to two fuzzy sets $A \& B$, total ambiguity may be defined as the sum of fuzzy information measure of $A$ and the fuzzy directed divergence measure of $A$ from $B$. It is not symmetric in nature. Verma and Sharma [5] defined the fuzzy inaccuracy measure corresponding to (1) as 


$$
I(A ; B)=-\frac{1}{n} \sum_{i=1}^{n}\left[\mu_{A}\left(x_{i}\right) \log \mu_{B}\left(x_{i}\right)+\left(1-\mu_{A}\left(x_{i}\right)\right) \log \left(1-\mu_{B}\left(x_{i}\right)\right)\right] .
$$

Hooda and Sharma [6] proposed the inaccuracy measure in the context of R-norm information measure (RIM) [7] as

$$
I_{R}(P ; Q)=\frac{R}{R-1}\left[\left(\sum_{i=1}^{n} p_{i}^{R} q_{i}^{1-R}\right)^{\frac{1}{R}}-\left(\sum_{i=1}^{n} p_{i}^{R}\right)^{\frac{1}{R}}\right] ; R>0(\neq 1)
$$

Then, Hooda and Bajaj [8] proposed the total ambiguity measure of (4) as

$$
I_{R}(A ; B)=\frac{R}{R-1}\left[\sum_{i=1}^{n}\left\{1-\left(\mu_{A}^{R}\left(x_{i}\right)+\left(1-\mu_{A}\left(x_{i}\right)\right)^{R}\right)^{\frac{1}{R}}\right\}+\sum_{i=1}^{n}\left\{\left(\mu_{A}^{R}\left(x_{i}\right) \mu_{B}^{1-R}\left(x_{i}\right)+\left(1-\mu_{A}\left(x_{i}\right)\right)^{R}\left(1-\mu_{B}\left(x_{i}\right)\right)^{1-R}\right)^{\frac{1}{R}}-1\right\}\right] ; R>0(\neq 1) .
$$

In the present paper, we have generalized various important measures of 'useful' R-norm inaccuracy and 'useful' total ambiguity that is shown in Sub-Section $C$ of Section II \& Sub-Section $B$ of Section III respectively. Further, the proposed 'useful' R-norm total ambiguity measure is successfully applied to MCDM technique.

Section wise break-up of the paper is described as: In the Section II the related work concerning the topic is given. This is followed by Section III in which we have proposed a new measure of 'useful' R-norm inaccuracy. Further, the properties, measure of information improvement, particular cases and the monotonic behaviour concerning the proposed measure are given in Sub-Sections $A, B, C$ and $D$ of Section III respectively. In Section IV, we have defined the fuzzy analogue of the measure presented in Section III along with its basic properties and particular cases that are shown in its subsequent Sub-Sections $A$ and $B$ respectively. Its Sub-Section $C$ pertains to the introduction of R-norm fuzzy information improvement measure. In Sub-Section $D$, we have studied the monotonic behaviour of the 'useful' $\mathrm{R}$-norm total ambiguity measure. In the last Sub-Section $E$ of IV, we have presented the application of 'useful' R-norm total ambiguity measure. Finally, in Section V, conclusion of the paper is provided.

\section{Related Work}

Recently, authors like Verma and Sharma [9] proposed fuzzy inaccuracy measure and studied its application in terms of MCDM, Bhat et al. [10] developed noiseless coding theorems for generalized 'useful' fuzzy inaccuracy measure and in the following year, Bhat et al. [11] characterized a new generalized inaccuracy measure alongwith its average code-word length. Further, many others have proposed different measures of inaccuracy for varying situations.

\section{Generalized 'Useful' R-Norm Inaccuracy Measure}

Consider the 'useful' RIM defined by Sofi et al. [12]

$$
H_{R}^{\alpha, \beta}(P ; U)=\frac{R+\alpha-\beta}{R-\beta}\left[1-\left\{\frac{\sum_{i=1}^{n} u_{i} p_{i}^{\frac{R+\alpha-\beta}{\alpha}}}{\sum_{i=1}^{n} u_{i} p_{i}}\right\}^{\frac{\alpha}{R+\alpha-\beta}}\right] ; R>0(\neq 1) ; 0<\alpha, \beta \leq 1 ; R \neq \beta ; u_{i}>0 \text {. }
$$

and the 'useful' R-norm directed divergence measure defined by Sofi et al. "unpublished" [13]

$$
D_{R}^{\alpha, \beta}(P: Q ; U)=\frac{R+\alpha-\beta}{\beta-R}\left[1-\left(\frac{\sum_{i=1}^{n} u_{i} p_{i}^{\frac{R+\alpha-\beta}{\alpha}} q_{i}^{1-\frac{R+\alpha-\beta}{\alpha}}}{\sum_{i=1}^{n} u_{i} p_{i}}\right)^{\frac{\alpha}{R+\alpha-\beta}}\right] ; R>0(\neq 1) ; 0<\alpha, \beta \leq 1 ; R>\beta \& u_{i}>0
$$

Corresponding to (6) and (7), we define the following 'useful' R-norm inaccuracy measure (RIAM) having two parameters $\alpha$ and $\beta$ : 
$I_{R}^{\alpha, \beta}(P ; Q ; U)=\frac{R+\alpha-\beta}{R-\beta}\left[\left(\frac{\sum_{i=1}^{n} u_{i} p_{i}^{\frac{R+\alpha-\beta}{\alpha}} q_{i}^{1-\frac{R+\alpha-\beta}{\alpha}}}{\sum_{i=1}^{n} u_{i} p_{i}}\right)^{\frac{\alpha}{R+\alpha-\beta}}-\left(\frac{\sum_{i=1}^{n} u_{i} p_{i}^{\frac{R+\alpha-\beta}{\alpha}}}{\sum_{i=1}^{n} u_{i} p_{i}}\right)^{\frac{\alpha}{R+\alpha-\beta}}\right] ; R>0(\neq 1) ; 0<\alpha, \beta \leq 1 ; R>\beta \& u_{i}>0$.

\subsection{Properties of 'Useful' RIAM (8)}

The 'useful' RIAM has the following properties:

1) Non-negativity i.e., $I_{R}^{\alpha, \beta}(P ; Q ; U) \geq 0$.

2) $H_{R}^{\alpha, \beta}(P ; U) \leq I_{R}^{\alpha, \beta}(P ; Q ; U)$.

3) $I_{R}^{\alpha, \beta}(P ; Q ; U)$ is symmetric function of its arguments.

4) $I_{R}^{\alpha, \beta}(P ; Q ; U)$ has an infinite value if $q_{i}=0, p_{i} \neq 0 \& u_{i} \neq 0$ for any $i$.

5) $I_{R}^{\alpha, \beta}(P ; Q ; U)$ has minimum value when $q_{i}=p_{i} \forall i$.

6) $I_{R}^{\alpha, \beta}(P ; Q ; U)=0$ if and only if $p_{i}=q_{i}=1$ for one value and $p_{i}=q_{i}=0$ for all other $i \& u_{i} \geq 0$.

With the help of following tables, the above properties are verified for the measure (8) by considering a hypothetical data.

Table 1. For Properties $1,2 \& 3$

\begin{tabular}{|c|c|c|c|c|c|c|c|c|}
\hline$p_{i}$ & $q_{i}$ & $u_{i}$ & $\alpha$ & $\beta$ & $R$ & $H_{R}^{\alpha, \beta}(P ; U)$ & $I_{R}^{\alpha, \beta}(P ; Q ; U)$ & $I_{R}^{\alpha, \beta}\left(P^{S} ; Q^{S} ; U^{S}\right)$ \\
\hline 0.13 & 0.23 & 5 & 0.23 & 0.34 & 0.65 & 0.9182 & 1.7569 & 1.7569 \\
\hline 0.03 & 0.11 & 2 & 0.45 & 0.51 & 70 & 1.1809 & 1.7127 & 1.7127 \\
\hline 0.41 & 0.17 & 4 & 0.20 & 0.20 & 11 & 0.6002 & 3.0417 & 3.0417 \\
\hline 0.15 & 0.30 & 1 & 0.92 & 0.85 & 100 & 0.5951 & 3.1142 & 3.1142 \\
\hline 0.18 & 0.05 & 3 & 0.88 & 0.27 & 140 & 0.5934 & 3.1382 & 3.1382 \\
\hline 0.10 & 0.14 & 6 & 0.15 & 0.95 & 13 & 0.5968 & 3.0890 & 3.0890 \\
\hline
\end{tabular}

From Table 1, it is clear that

1) $I_{R}^{\alpha, \beta}(P ; Q ; U)>0$.

2) $I_{R}^{\alpha, \beta}(P ; Q ; U)>H_{R}^{\alpha, \beta}(P ; U)$ and

3) The proposed 'useful' RIAM satisfies symmetry property, that is, $I_{R}^{\alpha, \beta}(P ; Q ; U)=I_{R}^{\alpha, \beta}\left(P^{s} ; Q^{s} ; U^{s}\right)$. Here, $I_{R}^{\alpha, \beta}\left(P^{s} ; Q^{s} ; U^{s}\right)$ represents the arrangement of elements of $I_{R}^{\alpha, \beta}(P ; Q ; U)$, in such a way that the one to one correspondence among the elements remains unchanged.

Table 2. Value of $I_{R}^{\alpha, \beta}(P ; Q ; U)$ when $q_{i}=0, p_{i} \neq 0 \& u_{i} \neq 0$ for $i=3$

\begin{tabular}{|c|c|c|c|c|c|c|}
\hline$p_{i}$ & $q_{i}$ & $u_{i}$ & $\alpha$ & $\beta$ & $R$ & $I_{R}^{\alpha, \beta}(P ; Q ; U)$ \\
\hline 0.13 & 0.23 & 5 & \multirow{2}{*}{0.23} & \multirow{2}{*}{0.34} & \multirow{2}{*}{0.65} & \multirow{2}{*}{$\infty$} \\
\hline 0.03 & 0.11 & 2 & & & & \\
\hline 0.41 & 0.00 & 4 & \multirow{2}{*}{0.45} & \multirow{2}{*}{0.51} & \multirow{2}{*}{70} & \multirow{2}{*}{$\infty$} \\
\hline 0.15 & 0.47 & 1 & & & & \\
\hline 0.18 & 0.05 & 3 & \multirow{2}{*}{0.92} & \multirow{2}{*}{0.85} & \multirow{2}{*}{100} & \multirow{2}{*}{$\infty$} \\
\hline 0.10 & 0.14 & 6 & & & & \\
\hline
\end{tabular}

It is clear from Table 2 that when $q_{i}=0$ for any $i$, (whatever be the values of $\alpha, \beta \& R$ ), we get $I_{R}^{\alpha, \beta}(P ; Q ; U)=\infty$. 
Table 3. For Property 5

\begin{tabular}{|c|c|c|c|c|c|c|c|c|}
\hline$p_{i}$ & $q_{i}$ & $u_{i}$ & $\alpha$ & $\beta$ & $R$ & $I_{R}^{\alpha, \beta}(P ; Q ; U)$ & $H_{R}^{\alpha, \beta}(P ; U)$ & $D_{R}^{\alpha, \beta}(P: Q ; U)$ \\
\hline 0.23 & 0.23 & 5 & \multirow{2}{*}{0.23} & \multirow{2}{*}{0.34} & \multirow{2}{*}{0.65} & \multirow{2}{*}{1.0726} & \multirow{2}{*}{1.0726} & \multirow{2}{*}{0.0} \\
\hline 0.11 & 0.11 & 2 & & & & & & \\
\hline 0.17 & 0.17 & 4 & \multirow{2}{*}{0.45} & \multirow{2}{*}{0.51} & \multirow{2}{*}{70} & \multirow{2}{*}{0.7068} & \multirow{2}{*}{0.7068} & \multirow{2}{*}{0.0} \\
\hline 0.30 & 0.30 & 1 & & & & & & \\
\hline 0.05 & 0.05 & 3 & \multirow{2}{*}{0.92} & \multirow{2}{*}{0.85} & \multirow{2}{*}{100} & \multirow{2}{*}{0.7098} & \multirow{2}{*}{0.7098} & \multirow{2}{*}{0.0} \\
\hline 0.14 & 0.14 & 6 & & & & & & \\
\hline
\end{tabular}

We can see from the Table 3 that when $q_{i}=p_{i} \forall i$, divergence term becomes zero and thus $I_{R}^{\alpha, \beta}(P ; Q ; U)=H_{R}^{\alpha, \beta}(P ; U)$. This gives the minimum value of $I_{R}^{\alpha, \beta}(P ; Q ; U)$.

Property 6: $I_{R}^{\alpha, \beta}(P ; Q ; U)=0$ if and only if $p_{i}=q_{i}=1$ for one value and $p_{i}=q_{i}=0 \forall i \& u_{i} \geq 0$.

Suppose $p_{i}=q_{i}=1$ for $i=1$ and for $i=2,3, \ldots, n ; p_{i}=q_{i}=0$, we have

$$
\begin{gathered}
I_{R}^{\alpha, \beta}(P ; Q ; U)=\frac{R+\alpha-\beta}{R-\beta}\left[\left(\frac{u_{1} p_{1}^{\frac{R+\alpha-\beta}{\alpha}} q_{1}^{1-\frac{R+\alpha-\beta}{\alpha}}}{u_{1} p_{1}}\right)^{\frac{\alpha}{R+\alpha-\beta}}-\left(\frac{u_{1} p_{1}^{\frac{R+\alpha-\beta}{\alpha}}}{u_{1} p_{1}}\right)^{\frac{\alpha}{R+\alpha-\beta}}\right]+\frac{R+\alpha-\beta}{R-\beta}\left[\left(\frac{\sum_{i=2}^{n} u_{i} p_{i}^{\frac{R+\alpha-\beta}{\alpha}} q_{i}^{1-\frac{R+\alpha-\beta}{\alpha}}}{\sum_{i=2}^{n} u_{i} p_{i}}\right)^{\frac{\alpha}{R+\alpha-\beta}}\right. \\
\left.-\left(\frac{\sum_{i=2}^{n} u_{i} p_{i}^{\frac{R+\alpha-\beta}{\alpha}}}{\sum_{i=2}^{n} u_{i} p_{i}}\right)^{\frac{\alpha}{R+\alpha-\beta}}\right] . \quad=\frac{R+\alpha-\beta}{R-\beta}\left[\left(\frac{u_{1}}{u_{1}}\right)^{\frac{\alpha}{R+\alpha-\beta}}-\left(\frac{u_{1}}{u_{1}}\right)^{\frac{\alpha}{R+\alpha-\beta}}\right]+0, \text { since } p_{1}=q_{1}=1 . \Rightarrow I_{R}^{\alpha, \beta}(P ; Q ; U)=0 .
\end{gathered}
$$

Hence, the result follows.

\subsection{Measure of Information Improvement}

The measure of information improvement (MII) was given by Theil [14] as

$$
D(P ; Q ; U)-D(P ; R ; U)
$$

where $P$ and $Q$ are the respective observed and predicted probability distributions of a random variable and $R$ represents the revised probability distribution of $Q$. Corresponding to the 'useful' R-norm DDM defined in (7), we define the following 'useful' R-norm MII as

$$
\begin{aligned}
& D_{R}^{\alpha, \beta}(P ; Q ; U)-D_{R}^{\alpha, \beta}(P ; R ; U) .=\frac{R+\alpha-\beta}{\beta-R}\left[1-\left(\frac{\sum_{i=1}^{n} u_{i} p_{i}^{\frac{R+\alpha-\beta}{\alpha}} q_{i}^{1-\frac{R+\alpha-\beta}{\alpha}}}{\sum_{i=1}^{n} u_{i} p_{i}}\right)^{\frac{\alpha}{R+\alpha-\beta}}\right] \\
& -\frac{R+\alpha-\beta}{\beta-R}\left[1-\left(\frac{\sum_{i=1}^{n} u_{i} p_{i}^{\frac{R+\alpha-\beta}{\alpha}} r_{i}^{1-\frac{R+\alpha-\beta}{\alpha}}}{\sum_{i=1}^{n} u_{i} p_{i}}\right)^{\frac{\alpha}{R+\alpha-\beta}}\right] ; R>0(\neq 1) ; 0<\alpha, \beta \leq 1 ; R>\beta \& u_{i}>0 .
\end{aligned}
$$

\subsection{Particular Cases of 'Useful' RIAM Defined in (8)}

- $\quad$ For $u_{i}=1$, the proposed measure (8) reduces to the RIAM defined by Peerzada et al. [15].. 
- $\quad$ For $\alpha=1, \beta=1 \& u_{i}=1$, the 'useful' RIAM (8) reduces to (4).

- $\quad$ For $\alpha=1, \beta=1 \& R \rightarrow 1$, the 'useful' RIAM (8) reduces to (2).

- $\quad$ For $\alpha=1, \beta=1, u_{i}=1 \& R \rightarrow 1$, 'useful' RIAM (8) reduces to (1).

\subsection{Monotone Behaviour of 'Useful' RIAM Defined in (8)}

We study the monotonic nature of the proposed measures in the given limits of $R, \alpha$ and $\beta$. We take two probability distributions $P \& Q: P=(0.41,0.13,0.10,0.18,0.15,0.03), Q=(0.23,0.05,0.14,0.30,0.17,0.11)$ with utility distribution $U=(5,2,4,1,3,6)$ and $n=6$. The results are given in the following tables by taking various values of $R$, $\alpha$ and $\beta$.

Table 4. Values of Measure (8) for Fixed $\alpha \& \beta$

\begin{tabular}{|c|c|c|c|c|c|c|c|c|}
\hline$R$ & 0.95 & 7 & 20 & 47 & 62 & 100 & 120 & 140 \\
\hline$I_{R}^{0.960 .20}(P ; Q ; U)$ & 1.3569 & 1.5086 & 1.8672 & 2.0447 & 2.0787 & 2.1201 & 2.1316 & 2.1398 \\
\hline$I_{R}^{0.59,0.59}(P ; Q ; U)$ & 1.3865 & 1.6502 & 1.9793 & 2.0983 & 2.1202 & 2.1465 & 2.1537 & 2.1589 \\
\hline$I_{R}^{0.620 .81}(P ; Q ; U)$ & 1.7115 & 2.2621 & 2.4266 & 2.4791 & 2.4886 & 2.5000 & 2.5030 & 2.5053 \\
\hline
\end{tabular}

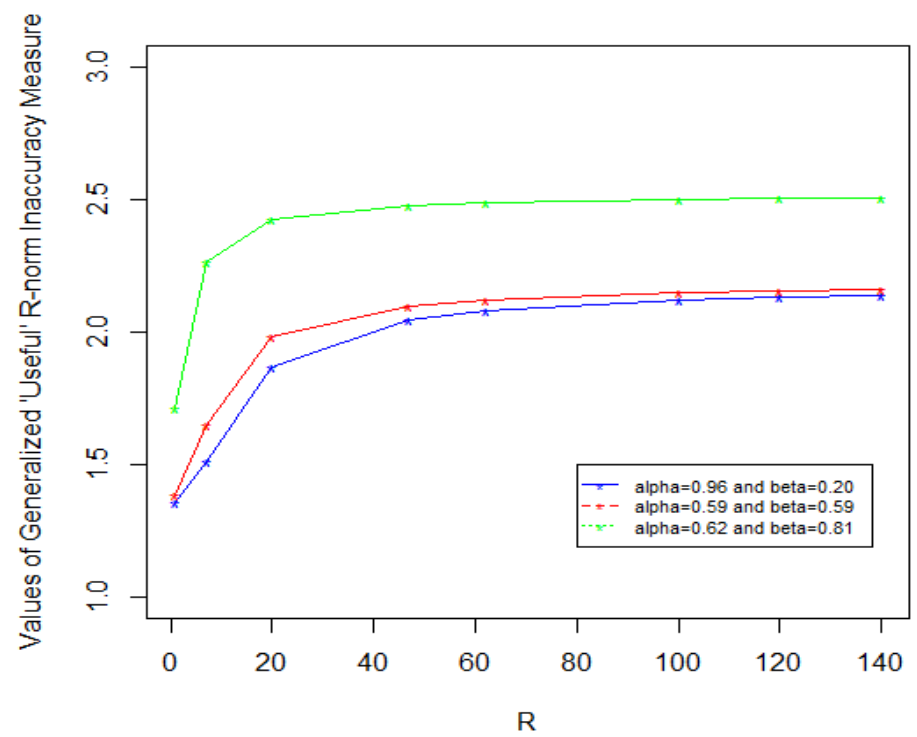

Fig.1. Graphical Overview of Measure (8) for Fixed Alpha and Beta

From Table 4, we can clearly see that as we increase the value of $R$ and keep $\alpha \& \beta$ fixed; the 'useful' RIAM defined in (8) shows an increasing trend. Although, the value of measure (8) changes if we alter the values of $\alpha \& \beta$ but the trend (that is increasing) remains the same. This increasing nature of measure (8) with respect to varying $R$ is depicted in the Fig. 1 by taking values of $\alpha \& \beta$ as $(0.96,0.20),(0.59,0.59) \&(0.62,0.81)$ respectively.

Table 5. Values of Measure (8) for Fixed $R$ and $\beta$

\begin{tabular}{|c|c|c|c|c|c|c|c|c|c|c|}
\hline$\alpha$ & 0.1 & 0.2 & 0.3 & 0.4 & 0.5 & 0.6 & 0.7 & 0.8 & 0.9 & 1.0 \\
\hline$I_{3}^{\alpha, 0.02}(P ; Q ; U)$ & 1.9590 & 1.7636 & 1.6182 & 1.5235 & 1.4623 & 1.4207 & 1.3910 & 1.3692 & 1.3528 & 1.3404 \\
\hline$I_{8}^{\alpha, 0.89}(P ; Q ; U)$ & 2.0889 & 1.9941 & 1.9051 & 1.8223 & 1.7472 & 1.6817 & 1.6261 & 1.5798 & 1.5415 & 1.5097 \\
\hline$I_{23}^{\alpha .052}(P ; Q ; U)$ & 2.1573 & 2.1253 & 2.0939 & 2.0631 & 2.0330 & 2.0035 & 1.9745 & 1.9461 & 1.9183 & 1.8911 \\
\hline
\end{tabular}




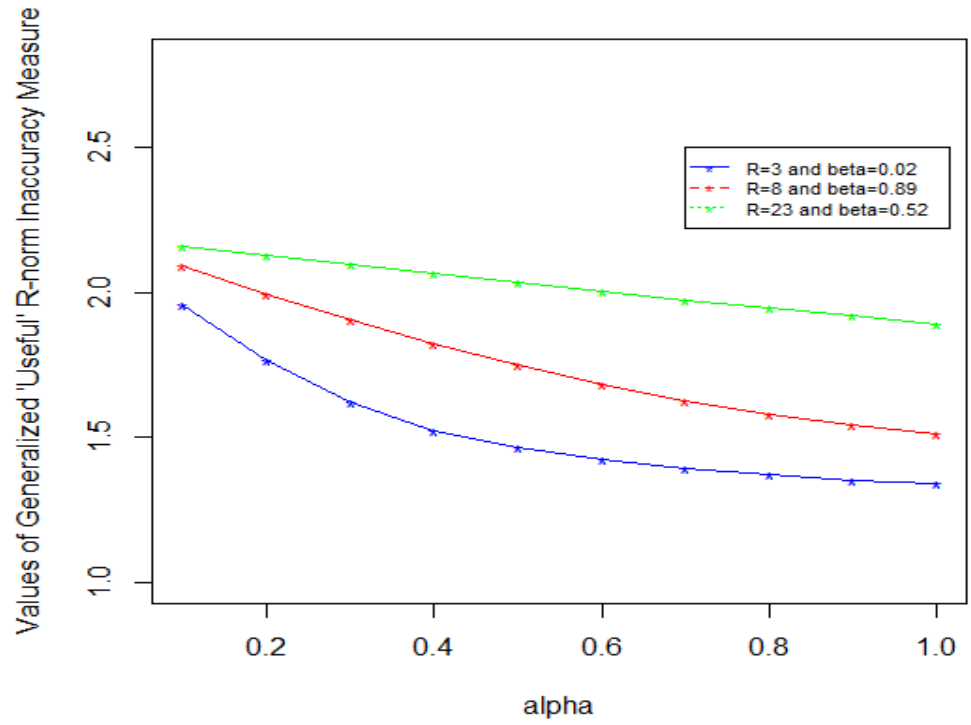

Fig.2. Graphical Overview of Measure (8) for Fixed $R$ and Beta

From Table 5, we can easily state that as the value of $\alpha$ increases ( $R$ and $\beta$ are fixed), measure (8) decreases. This relation exists for different possible values of $R$ and $\beta$. Thus, there is a negative relation between $\alpha$ and the measure (8). This relation is depicted in the Fig. 2 by taking values of $R$ and $\beta$ as $(3,0.02),(8,0.89) \&(23,0.52)$ respectively.

Table 6. For Fixed $R$ and $\alpha$

\begin{tabular}{|c|c|c|c|c|c|c|c|c|c|c|}
\hline$\beta$ & 0.1 & 0.2 & 0.3 & 0.4 & 0.5 & 0.6 & 0.7 & 0.8 & 0.9 & 1.0 \\
\hline$I_{2}^{0.12, \beta}(P ; Q ; U)$ & 1.7844 & 1.7659 & 1.7459 & 1.7243 & 1.7011 & 1.6760 & 1.6492 & 1.6206 & 1.5903 & 1.5585 \\
\hline$I_{11}^{0.15, \beta}(P ; Q ; U)$ & 1.8772 & 1.8746 & 1.8720 & 1.8693 & 1.8666 & 1.8639 & 1.8611 & 1.8582 & 1.8553 & 1.8524 \\
\hline$I_{23}^{0.98, \beta}(P ; Q ; U)$ & 1.9014 & 1.9002 & 1.8991 & 1.8979 & 1.8968 & 1.8955 & 1.8943 & 1.8931 & 1.8919 & 1.8907 \\
\hline
\end{tabular}

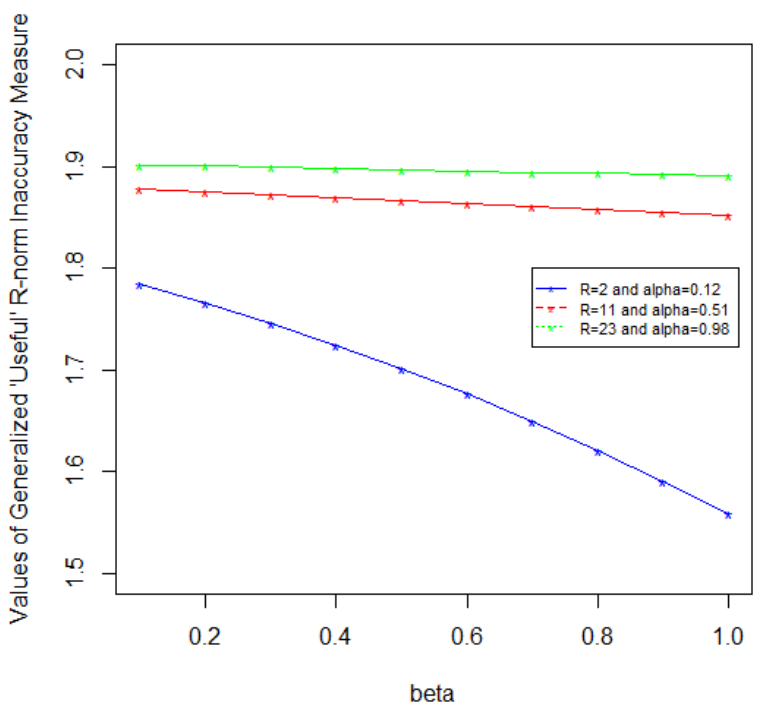

Fig.3. Graphical Overview of Measure (8) for Fixed $R$ and Beta

From Table 6, we can easily state that as the value of $\beta$ increases ( $R$ and $\alpha$ are fixed), the value of measure (8) decreases. Thus there is a negative relation between $\beta$ and the measure (8). This relation is depicted in the Fig. 3 by taking values of $R$ and $\alpha$ as $(2,0.12),(11,0.51) \&(23,0.98)$ respectively. 


\section{Generalized Measure of 'Useful' R-Norm Total Ambiguity}

Consider the 'useful' RFIM defined by Sofi et al. [16]

$$
H_{R}^{\alpha, \beta}(A ; U)=\frac{R+\alpha-\beta}{R-\beta}\left[\frac{n \sum_{i=1}^{n} u_{i}\left[1-\left\{\mu_{A}^{\frac{R+\alpha-\beta}{\alpha}}\left(x_{i}\right)+\left(1-\mu_{A}\left(x_{i}\right)\right)^{\frac{R+\alpha-\beta}{\alpha}}\right\}^{\frac{\alpha}{R+\alpha-\beta}}\right]}{\sum_{i=1}^{n} u_{i}}\right] ; R>0(\neq 1) ; 0<(\alpha, \beta) \leq 1 \& u_{i}>0
$$

and the 'useful' R-norm fuzzy directed divergence measure defined by Sofi et al. "unpublished" [13]:

$$
\begin{gathered}
D_{R}^{\alpha, \beta}(A, B ; U)=\frac{R+\alpha-\beta}{\beta-R}\left[\frac{n \sum_{i=1}^{n} u_{i}}{\sum_{i=1}^{n} u_{i}}\left\{1-\left(\mu_{A}^{\frac{R+\alpha-\beta}{\alpha}}\left(x_{i}\right) \mu_{B}^{1-\frac{R+\alpha-\beta}{\alpha}}\left(x_{i}\right)+\left(1-\mu_{A}\left(x_{i}\right)\right)^{\frac{R+\alpha-\beta}{\alpha}}\left(1-\mu_{B}\left(x_{i}\right)\right)^{1-\frac{R+\alpha-\beta}{\alpha}}\right)^{\frac{\alpha}{R+\alpha-\beta}}\right\} ;\right. \\
R>0(\neq 1) ; 0<\alpha, \beta \leq 1 ;(R+\alpha)>\beta \& u_{i}>0 .
\end{gathered}
$$

Corresponding to (12) and (13), we define the following 'useful' R-norm total ambiguity (or fuzzy inaccuracy) measure (RTAM):

$$
\begin{aligned}
& I_{R}^{\alpha, \beta}(A ; B ; U)=n \frac{R+\alpha-\beta}{R-\beta}\left[\frac{\sum_{i=1}^{n} u_{i}}{\sum_{i=1}^{n} u_{i}}\left\{\left(\mu_{A}^{\frac{R+\alpha-\beta}{\alpha}}\left(x_{i}\right) \mu_{B}^{1-\frac{R+\alpha-\beta}{\alpha}}\left(x_{i}\right)+\left(1-\mu_{A}\left(x_{i}\right)\right)^{\frac{R+\alpha-\beta}{\alpha}}\left(1-\mu_{B}\left(x_{i}\right)\right)^{1-\frac{R+\alpha-\beta}{\alpha}}\right)^{\frac{\alpha}{R+\alpha-\beta}}\right\}\right. \\
& \left.-\frac{\sum_{i=1}^{n} u_{i}\left\{\left(\mu_{A}^{\frac{R+\alpha-\beta}{\alpha}}\left(x_{i}\right)+\left(1-\mu_{A}\left(x_{i}\right)\right)^{\frac{R+\alpha-\beta}{\alpha}}\right)^{\frac{\alpha}{R+\alpha-\beta}}\right\}}{\sum_{i=1}^{n} u_{i}}\right] ; R>0(\neq 1) ; 0<\alpha, \beta \leq 1 ;(R+\alpha)>\beta \& u_{i}>0 .
\end{aligned}
$$

\subsection{Properties of 'Useful' RTAM (14)}

1) $I_{R}^{\alpha, \beta}(A ; B ; U)=0$ if and only if either $\mu_{A}\left(x_{i}\right)=\mu_{B}\left(x_{i}\right)=0$ or $\mu_{A}\left(x_{i}\right)=\mu_{B}\left(x_{i}\right)=1 \quad \forall x_{i} \in X ; i=1,2, \ldots, n$.

2) $I_{R}^{\alpha, \beta}(A ; B ; U)>0$.

3) $I_{R}^{\alpha, \beta}(A ; B ; U)$ is a symmetric function of its arguments.

4) For any two fuzzy sets $A \& B, I_{R}^{\alpha, \beta}(A ; B ; U) \geq H_{R}^{\alpha, \beta}(A ; U)$ with equality if and only if $\mu_{A}\left(x_{i}\right)=\mu_{B}\left(x_{i}\right)$.

For property 1, let's assume $\mu_{A}\left(x_{i}\right)=\mu_{B}\left(x_{i}\right)=0$. Thus, we have

$$
I_{R}^{\alpha, \beta}(A ; B ; U)=n \frac{R+\alpha-\beta}{R-\beta}\left[\frac{\sum_{i=1}^{n} u_{i}\left\{(0+1)^{\left.\frac{\alpha}{R+\alpha-\beta}\right\}}\right.}{\sum_{i=1}^{n} u_{i}}-\frac{\sum_{i=1}^{n} u_{i}\left\{\left(0+(1)^{\frac{R+\alpha-\beta}{\alpha}}\right)\right\}^{\frac{\alpha}{R+\alpha-\beta}}}{\sum_{i=1}^{n} u_{i}}\right]=n \frac{R+\alpha-\beta}{R-\beta}\left[\frac{\sum_{i=1}^{n} u_{i}}{\sum_{i=1}^{n} u_{i}}-\frac{\sum_{i=1}^{n} u_{i}}{\sum_{i=1}^{n} u_{i}}\right] .=0 .
$$

Similarly, if $\mu_{A}\left(x_{i}\right)=\mu_{B}\left(x_{i}\right)=1$, then $I_{R}^{\alpha, \beta}(A ; B ; U)=0$.

Conversely, suppose $I_{R}^{\alpha, \beta}(A ; B ; U)=0$, then 


$$
\begin{aligned}
& {\left[\frac{\sum_{i=1}^{n} u_{i}\left\{\left(\mu_{A}^{\frac{R+\alpha-\beta}{\alpha}}\left(x_{i}\right) \mu_{B}^{1-\frac{R+\alpha-\beta}{\alpha}}\left(x_{i}\right)+\left(1-\mu_{A}\left(x_{i}\right)\right)^{\frac{R+\alpha-\beta}{\alpha}}\left(1-\mu_{B}\left(x_{i}\right)\right)^{1-\frac{R+\alpha-\beta}{\alpha}}\right)^{\frac{\alpha}{R+\alpha-\beta}}\right\}}{\sum_{i=1}^{n} u_{i}}-\frac{\sum_{i=1}^{n} u_{i}\left\{\left(\mu_{A}^{\frac{R+\alpha-\beta}{\alpha}}\left(x_{i}\right)+\left(1-\mu_{A}\left(x_{i}\right)\right)^{\frac{R+\alpha-\beta}{\alpha}}\right)^{\frac{\alpha}{R+\alpha-\beta}}\right\}}{\sum_{i=1}^{n} u_{i}}\right]=0 .} \\
& \Rightarrow \sum_{i=1}^{n} u_{i}\left\{\left(\mu_{A}^{\frac{R+\alpha-\beta}{\alpha}}\left(x_{i}\right) \mu_{B}^{1-\frac{R+\alpha+\beta-\beta}{\alpha}}\left(x_{i}\right)+\left(1-\mu_{A}\left(x_{i}\right)\right)^{\frac{R+\alpha-\beta}{\alpha}}\left(1-\mu_{B}\left(x_{i}\right)\right)^{\frac{1-\frac{R+\alpha-\beta}{\alpha}}{\alpha}}\right)^{\frac{\alpha}{R+\alpha-\beta}}\right\}=\sum_{i=1}^{n} u_{i}\left\{\left(\mu_{A}^{\frac{R+\alpha-\beta}{\alpha}}\left(x_{i}\right)+\left(1-\mu_{A}\left(x_{i}\right)\right)^{\frac{R+\alpha-\beta}{\alpha}}\right)^{\frac{\alpha}{R+\alpha-\beta}}\right\} .
\end{aligned}
$$

The relation (16) holds only if $\mu_{A}\left(x_{i}\right)=\mu_{B}\left(x_{i}\right)=0$ or $\mu_{A}\left(x_{i}\right)=\mu_{B}\left(x_{i}\right)=1$.

Hence property 1 is proved.

The properties 2, 3 and 4 for the measure (14) are verified with the help of following tables by considering a hypothetical data.

Table 7. For Verification of Properties 2 and 3

\begin{tabular}{|c|c|c|c|c|c|c|c|c|}
\hline$\mu_{A}\left(x_{i}\right)$ & $\mu_{B}\left(x_{i}\right)$ & $\mu_{i}$ & $\alpha$ & $\beta$ & $R$ & $H_{R}^{\alpha, \beta}(A ; U)$ & $I_{R}^{\alpha, \beta}(A ; B ; U)$ & $I_{R}^{\alpha, \beta}\left(A^{s} ; B^{s} ; U^{s}\right)$ \\
\hline 0.65 & 0.42 & 5 & 0.23 & 0.34 & 0.65 & 2.2702 & 11.2197 & 11.2197 \\
\hline 0.23 & 0.28 & 2 & 0.45 & 0.51 & 70 & 1.5298 & 22.5865 & 22.5865 \\
\hline 0.82 & 0.05 & 4 & 0.20 & 0.20 & 11 & 1.5481 & 22.1032 & 22.1032 \\
\hline 0.44 & 0.90 & 1 & 0.92 & 0.85 & 100 & 1.5341 & 22.4718 & 22.4718 \\
\hline 0.97 & 0.73 & 3 & 0.88 & 0.27 & 140 & 1.5295 & 22.5938 & 22.5938 \\
\hline 0.31 & 0.61 & 6 & 0.15 & 0.95 & 13 & 1.5389 & 22.3438 & 22.3438 \\
\hline
\end{tabular}

From Table 7, it is clear that

- $I_{R}^{\alpha, \beta}(A ; B ; U)>0$.

- $\quad I_{R}^{\alpha, \beta}(A ; B ; U)>H_{R}^{\alpha, \beta}(A ; U)$ and

- The proposed 'useful' RTAM satisfies symmetry property, that is, $I_{R}^{\alpha, \beta}(A ; B ; U)=I_{R}^{\alpha, \beta}\left(A^{s} ; B^{s} ; U^{s}\right)$. Here, $I_{R}^{\alpha, \beta}\left(A^{s} ; B^{s} ; U^{s}\right)$ represents the arrangement of elements of $I_{R}^{\alpha, \beta}(A ; B ; U)$, in such a way that the one to one correspondence among the elements remains unchanged.

Table 8. Value of $I_{R}^{\alpha, \beta}(A ; B ; U)$ when $\mu_{A}\left(x_{i}\right)=\mu_{B}\left(x_{i}\right)$

\begin{tabular}{|c|c|c|c|c|c|c|c|c|}
\hline$\mu_{A}\left(x_{i}\right)$ & $\mu_{B}\left(x_{i}\right)$ & $\mu_{i}$ & $\alpha$ & $\beta$ & $R$ & $H_{R}^{\alpha, \beta}(A ; U)$ & $I_{R}^{\alpha, \beta}(A ; B ; U)$ & $D_{R}^{\alpha, \beta}(A ; B ; U)$ \\
\hline 0.65 & 0.65 & 5 & 0.23 & 0.34 & 0.65 & 2.2702 & 2.2702 & 0.0 \\
\hline 0.23 & 0.23 & 2 & 0.45 & 0.51 & 70 & 1.5298 & 1.5298 & 0.0 \\
\hline 0.82 & 0.82 & 4 & 0.20 & 0.20 & 11 & 1.5481 & 1.5481 & 0.0 \\
\hline 0.44 & 0.44 & 1 & 0.92 & 0.85 & 100 & 1.5341 & 1.5341 & 0.0 \\
\hline 0.97 & 0.97 & 3 & 0.88 & 0.27 & 140 & 1.5295 & 1.5295 & 0.0 \\
\hline 0.31 & 0.31 & 6 & 0.15 & 0.95 & 13 & 1.5389 & 1.5389 & 0.0 \\
\hline
\end{tabular}

From Table 8, we conclude that when $\mu_{A}\left(x_{i}\right)=\mu_{B}\left(x_{i}\right), I_{R}^{\alpha, \beta}(A ; B ; U)=H_{R}^{\alpha, \beta}(A ; U)$ and the error term vanishes.

\subsection{Particular Cases of 'Useful' RTAM (14)}

- For $u_{i}=1$, the 'useful' RTAM (14) reduces to the R-norm fuzzy inaccuracy measure defined by Peerzada et al. [15].

- For $\alpha=1, \beta=1 \& u_{i}=1$, the 'useful' RTAM (14) reduces to (5).

- For $\alpha=1, \beta=1, u_{i}=1 \& R \rightarrow 1$, the 'useful' RTAM (14) reduces to (3).

Theorem I

1) $I_{R}^{\alpha, \beta}(A \cup B ; A \cap B ; U)+I_{R}^{\alpha, \beta}(A \cap B ; A \cup B ; U)$

$$
=I_{R}^{\alpha, \beta}(A ; B ; U)+I_{R}^{\alpha, \beta}(B ; A ; U) .
$$


2) $I_{R}^{\alpha, \beta}(A \cup B ; C ; U)+I_{R}^{\alpha, \beta}(A \cap B ; C ; U)$

$$
=I_{R}^{\alpha, \beta}(A ; C ; U)+I_{R}^{\alpha, \beta}(B ; C ; U) .
$$

3) $I_{R}^{\alpha, \beta}(A ; B \cup C ; U)+I_{R}^{\alpha, \beta}(A ; B \cap C ; U)$

$$
=I_{R}^{\alpha, \beta}(A ; B ; U)+I_{R}^{\alpha, \beta}(A ; C ; U) .
$$

For proving theorem I, we define $\mathrm{X}=\left\{x_{1}, x_{2}, \ldots, x_{n}\right\}$ as universe of discourse. Any fuzzy set $A$ is defined as $A=\left\{\left(x_{i}, \mu_{A}\left(x_{i}\right)\right) ; x_{i} \in \mathrm{X}\right\}$ where $\mu_{A}\left(x_{i}\right)$ represents the membership function of $A$.

$A \cup B \& A \cap B$ are defined as:

- $A \cup B=\left\{\left\langle x_{i}, \mu_{A}\left(x_{i}\right) \Delta \mu_{B}\left(x_{i}\right)\right\rangle ; x_{i} \in \mathrm{X}\right\}$.

- $A \cap B=\left\{\left\langle x_{i}, \mu_{A}\left(x_{i}\right) \nabla \mu_{B}\left(x_{i}\right)\right\rangle ; x_{i} \in \mathrm{X}\right\}$.

where $\Delta \& \nabla$ respectively represent the maximum and minimum operators.

Also, assume $t=\frac{R+\alpha-\beta}{R-\beta} ; r=\frac{R+\alpha-\beta}{\alpha} ; x_{A}=\mu_{A}\left(x_{i}\right) ; x_{B}=\mu_{B}\left(x_{i}\right) \& x_{C}=\mu_{C}\left(x_{i}\right)$. Also, $X$ is separated in two parts $X_{1}$ and $X_{2}$ as

$$
\mathrm{X}_{1}=\left\{x: x_{i} \in \mathrm{X}, \mu_{A}\left(x_{i}\right) \geq \mu_{B}\left(x_{i}\right)\right\} \& \mathrm{X}_{2}=\left\{x: x_{i} \in \mathrm{X}, \mu_{A}\left(x_{i}\right)<\mu_{B}\left(x_{i}\right)\right\}
$$

Thus, we can write (14) as:

$$
I_{R}^{\alpha, \beta}(A ; B ; U)=n t\left[\frac{\sum_{i=1}^{n} u_{i}\left\{\left(x_{A}^{r} x_{B}^{1-r}+\left(1-x_{A}\right)^{r}\left(1-x_{B}\right)^{1-r}\right)^{\frac{1}{r}}\right\}}{\sum_{i=1}^{n} u_{i}}-\frac{\sum_{i=1}^{n} u_{i}\left\{\left(x_{A}^{r}+\left(1-x_{A}\right)^{r}\right)^{\frac{1}{r}}\right\}}{\sum_{i=1}^{n} u_{i}}\right] .
$$

Now,

1) $I_{R}^{\alpha, \beta}(A \cup B ; A \cap B ; U)+I_{R}^{\alpha, \beta}(A \cap B ; A \cup B ; U)=I_{R}^{\alpha, \beta}(A ; B ; U)+I_{R}^{\alpha, \beta}(B ; A ; U)$.

Proof: Consider

$$
\begin{aligned}
I_{R}^{\alpha, \beta}(A \cup B ; A \cap B ; & =n t\left[\frac{\sum_{i=1}^{n} u_{i}\left\{\left(x_{A \cup B}^{r} x_{A \cap B}^{1-r}+\left(1-x_{A \cup B}\right)^{r}\left(1-x_{A \cap B}\right)^{1-r}\right)^{\frac{1}{r}}\right\}}{\sum_{i=1}^{n} u_{i}}-\frac{\sum_{i=1}^{n} u_{i}\left\{\left(x_{A \cup B}^{r}+\left(1-x_{A \cup B}\right)^{r}\right)^{\frac{1}{r}}\right\}}{\sum_{i=1}^{n} u_{i}}\right] . \\
= & n t\left[\frac{\sum_{x_{i} \in X_{1}} u_{i}\left\{\left(x_{A}^{r} x_{B}^{1-r}+\left(1-x_{A}\right)^{r}\left(1-x_{B}\right)^{1-r}\right)^{\frac{1}{r}}\right\}}{\sum_{x_{i} \in X_{1}} u_{i}}-\frac{\sum_{x_{i} \in X_{1}} u_{i}\left\{\left(x_{A}^{r}+\left(1-x_{A}\right)^{r}\right)^{\frac{1}{r}}\right\}}{\sum_{x_{i} \in X_{1}} u_{i}}\right] \\
& +n t\left[\frac{\sum_{x_{i} \in X_{2}} u_{i}\left\{\left(x_{B}^{r} x_{A}^{1-r}+\left(1-x_{B}\right)^{r}\left(1-x_{A}\right)^{1-r}\right)^{\frac{1}{r}}\right\}}{\sum_{x_{i} X_{2}} u_{i}}-\frac{\sum_{x_{i} \in X_{2}} u_{i}\left\{\left(x_{B}^{r}+\left(1-x_{B}\right)^{r}\right)^{\frac{1}{r}}\right\}}{\sum_{x_{i} \in X_{2}} u_{i}}\right] .
\end{aligned}
$$

Now, 


$$
\begin{aligned}
I_{R}^{\alpha, \beta}(A \cap B ; A \cup B ; U)=n t\left[\frac{\sum_{i=1}^{n} u_{i}\left\{\left(x_{A \cap B}^{r} x_{A \cup B}^{1-r}+\left(1-x_{A \cap B}\right)^{r}\left(1-x_{A \cup B}\right)^{1-r}\right)^{\frac{1}{r}}\right\}}{\sum_{i=1}^{n} u_{i}}-\frac{\sum_{i=1}^{n} u_{i}\left\{\left(x_{A \cap B}^{r}+\left(1-x_{A \cap B}\right)^{r}\right)^{\frac{1}{r}}\right\}}{\sum_{i=1}^{n} u_{i}}\right] . \\
=n t\left[\frac{\sum_{x_{i} \in X_{1}} u_{i}\left\{\left(x_{B}^{r} x_{A}^{1-r}+\left(1-x_{B}\right)^{r}\left(1-x_{A}\right)^{1-r}\right)^{\frac{1}{r}}\right\}}{\sum_{x_{i} \in X_{i}} u_{i}}-\frac{\sum_{x_{i} \in X_{1}} u_{i}\left\{\left(x_{B}^{r}+\left(1-x_{B}\right)^{r}\right)^{\frac{1}{r}}\right\}}{\sum_{x_{i} \in X_{i}} u_{i}}\right] \\
+n t\left[\frac{\sum_{x_{i} \in X_{2}} u_{i}\left\{\left(x_{A}^{r} x_{B}^{1-r}+\left(1-x_{A}\right)^{r}\left(1-x_{B}\right)^{1-r}\right)^{\frac{1}{r}}\right\}}{\sum_{x_{i} \in X_{2}} u_{i}}-\frac{\sum_{x_{i} \in X_{2}} u_{i}\left\{\left(x_{A}^{r}+\left(1-x_{A}\right)^{r}\right)^{\frac{1}{r}}\right\}}{\sum_{x_{i} \in X_{2}} u_{i}}\right] .
\end{aligned}
$$

Adding (22) and (23), we get

$$
\begin{array}{r}
I_{R}^{\alpha, \beta}(A \cup B ; A \cap B ; U)+I_{R}^{\alpha, \beta}(A \cap B ; A \cup B ; U)=n t\left[\frac{\sum_{i=1}^{n} u_{i}\left\{\left(x_{A}^{r} x_{B}^{1-r}+\left(1-x_{A}\right)^{r}\left(1-x_{B}\right)^{1-r}\right)^{\frac{1}{r}}\right\}}{\sum_{i=1}^{n} u_{i}}-\frac{\sum_{i=1}^{n} u_{i}\left\{\left(x_{A}^{r}+\left(1-x_{A}\right)^{r}\right)^{\frac{1}{r}}\right\}}{\sum_{i=1}^{n} u_{i}}\right] \\
+n t\left[\frac{\sum_{i=1}^{n} u_{i}\left\{\left(x_{B}^{r} x_{A}^{1-r}+\left(1-x_{B}\right)^{r}\left(1-x_{A}\right)^{1-r}\right)^{\frac{1}{r}}\right\}}{\sum_{i=1}^{n} u_{i}}-\frac{\sum_{i=1}^{n} u_{i}\left\{\left(x_{B}^{r}+\left(1-x_{B}\right)^{r}\right)^{\frac{1}{r}}\right\}}{\sum_{i=1}^{n} u_{i}}\right] .=I_{R}^{\alpha, \beta}(A ; B ; U)+I_{R}^{\alpha, \beta}(B ; A ; U) .
\end{array}
$$

Hence, the result.

2) $I_{R}^{\alpha, \beta}(A \cup B ; C ; U)+I_{R}^{\alpha, \beta}(A \cap B ; C ; U)=I_{R}^{\alpha, \beta}(A ; C ; U)+I_{R}^{\alpha, \beta}(B ; C ; U)$

Proof: Consider

$$
\begin{aligned}
I_{R}^{\alpha, \beta}(A \cup B ; C ; U)+ & I_{R}^{\alpha, \beta}(A \cap B ; C ; U)=n t\left[\frac{\sum_{i=1}^{n} u_{i}\left\{\left(x_{A \cup B}^{r} x_{C}^{1-r}+\left(1-x_{A \cup B}\right)^{r}\left(1-x_{C}\right)^{1-r}\right)^{\frac{1}{r}}\right\}}{\sum_{i=1}^{n} u_{i}}-\frac{\sum_{i=1}^{n} u_{i}\left\{\left(x_{A \cup B}^{r}+\left(1-x_{A \cup B}\right)^{r}\right)^{\frac{1}{r}}\right\}}{\sum_{i=1}^{n} u_{i}}\right] \\
+ & n t\left[\frac{\sum_{i=1}^{n} u_{i}\left\{\left(x_{A \cap B}^{r} x_{C}^{1-r}+\left(1-x_{A \cap B}\right)^{r}\left(1-x_{C}\right)^{1-r}\right)^{\frac{1}{r}}\right\}}{\sum_{i=1}^{n} u_{i}}-\frac{\sum_{i=1}^{n} u_{i}\left\{\left(x_{A \cap B}^{r}+\left(1-x_{A \cap B}\right)^{r}\right)^{\frac{1}{r}}\right\}}{\sum_{i=1}^{n} u_{i}}\right] . \\
& =\left\{n t\left[\frac{\sum_{x_{i} \in X_{1}} u_{i}\left\{\left(x_{A}^{r} x_{C}^{1-r}+\left(1-x_{A}\right)^{r}\left(1-x_{C}\right)^{1-r}\right)^{\frac{1}{r}}\right\}}{\sum_{x_{i} \in \mathrm{X}_{1}} u_{i}}-\frac{\sum_{x_{i} \in X_{1}} u_{i}\left\{\left(x_{A}^{r}+\left(1-x_{A}\right)^{r}\right)^{r}\right\}}{\sum_{x_{i} \in X_{1}} u_{i}}\right]\right. \\
+ & \left.n t\left[\frac{\sum_{x_{i} \in X_{2}} u_{i}\left\{\left(x_{B}^{r} x_{C}^{1-r}+\left(1-x_{B}\right)^{r}\left(1-x_{C}\right)^{1-r}\right)^{\frac{1}{r}}\right\}}{\sum_{x_{i} \in \mathrm{X}_{2}} u_{i}}-\frac{\sum_{x_{i} \in X_{2}} u_{i}\left\{\left(x_{B}^{r}+\left(1-x_{B}\right)^{r}\right)^{\frac{1}{r}}\right\}}{\sum_{x_{i} \in X_{2}} u_{i}}\right]\right\}
\end{aligned}
$$




$$
\begin{gathered}
+\left\{n t\left[\frac{\sum_{x_{i} \in \mathrm{X}_{1}} u_{i}\left\{\left(x_{B}^{r} x_{C}^{1-r}+\left(1-x_{B}\right)^{r}\left(1-x_{C}\right)^{1-r}\right)^{\frac{1}{r}}\right\}}{\sum_{x_{i} \in \mathrm{X}_{i}} u_{i}}-\frac{\sum_{x_{i} \in \mathrm{X}_{1}} u_{i}\left\{\left(x_{B}^{r}+\left(1-x_{B}\right)^{r}\right)^{\frac{1}{r}}\right\}}{\sum_{x_{i} \in \mathrm{X}_{1}} u_{i}}\right]\right. \\
\left.+\left[\frac{\sum_{x_{i} \in \mathrm{X}_{2}} u_{i}\left\{\left(x_{A}^{r} x_{C}^{1-r}+\left(1-x_{A}\right)^{r}\left(1-x_{C}\right)^{1-r}\right)^{\frac{1}{r}}\right\}}{\sum_{x_{i} \in \mathrm{X}_{2}} u_{i}}-\frac{\sum_{x_{i} \in \mathrm{X}_{2}} u_{i}\left\{\left(x_{A}^{r}+\left(1-x_{A}\right)^{r}\right)^{\frac{1}{r}}\right\}}{\sum_{x_{i} \in \mathrm{X}_{2}} u_{i}}\right]\right\} \\
=n t\left[\frac{\sum_{i=1}^{n} u_{i}\left\{\left(x_{A}^{r} x_{C}^{1-r}+\left(1-x_{A}\right)^{r}\left(1-x_{C}\right)^{1-r}\right)^{\frac{1}{r}}\right\}}{\sum_{i=1}^{n} u_{i}}-\frac{\sum_{i=1}^{n} u_{i}\left\{\left(x_{A}^{r}+\left(1-x_{A}\right)^{r}\right)^{\frac{1}{r}}\right\}}{\sum_{i=1}^{n} u_{i}}\right] \\
+n t\left[\frac{\sum_{i=1}^{n} u_{i}\left\{\left(x_{B}^{r} x_{C}^{1-r}+\left(1-x_{B}\right)^{r}\left(1-x_{C}\right)^{1-r}\right)^{\frac{1}{r}}\right\}}{\sum_{i=1}^{n} u_{i}}-\frac{\sum_{i=1}^{n} u_{i}\left\{\left(x_{B}^{r}+\left(1-x_{B}\right)^{r}\right)^{\frac{1}{r}}\right\}}{\sum_{i=1}^{n} u_{i}}\right] . \\
=I_{R}^{\alpha, \beta}(A ; C ; U)+I_{R}^{\alpha, \beta}(B ; C ; U) .
\end{gathered}
$$

Hence, the result.

3) $I_{R}^{\alpha, \beta}(A ; B \cup C ; U)+I_{R}^{\alpha, \beta}(A ; B \cap C ; U)=I_{R}^{\alpha, \beta}(A ; B ; U)+I_{R}^{\alpha, \beta}(A ; C ; U)$

Consider

$$
\begin{aligned}
& I_{R}^{\alpha, \beta}(A ; B \cup C ; U)+I_{R}^{\alpha, \beta}(A ; B \cap C ; U)=n t\left[\frac{\sum_{i=1}^{n} u_{i}\left\{\left(x_{A}^{r} x_{B \cup C}^{1-r}+\left(1-x_{A}\right)^{r}\left(1-x_{B \cup C}\right)^{1-r}\right)^{\frac{1}{r}}\right\}}{\sum_{i=1}^{n} u_{i}}-\frac{\sum_{i=1}^{n} u_{i}\left\{\left(x_{A}^{r}+\left(1-x_{A}\right)^{r}\right)^{\frac{1}{r}}\right\}}{\sum_{i=1}^{n} u_{i}}\right] \\
& +n t\left[\frac{\sum_{i=1}^{n} u_{i}\left\{\left(x_{A}^{r} x_{B \cap C}^{1-r}+\left(1-x_{A}\right)^{r}\left(1-x_{B \cap C}\right)^{1-r}\right)^{\frac{1}{r}}\right\}}{\sum_{i=1}^{n} u_{i}}-\frac{\sum_{i=1}^{n} u_{i}\left\{\left(x_{A}^{r}+\left(1-x_{A}\right)^{r}\right)^{\frac{1}{r}}\right\}}{\sum_{i=1}^{n} u_{i}}\right] \\
& =\left\{n t\left[\frac{\sum_{x_{i} \in X_{1}} u_{i}\left\{\left(x_{A}^{r} x_{B}^{1-r}+\left(1-x_{A}\right)^{r}\left(1-x_{B}\right)^{1-r}\right)^{\frac{1}{r}}\right\}}{\sum_{x_{i} \in X_{i}} u_{i}}-\frac{\sum_{x_{i} \in X_{1}} u_{i}\left\{\left(x_{A}^{r}+\left(1-x_{A}\right)^{r}\right)^{\frac{1}{r}}\right\}}{\sum_{x_{i} \in X_{i}} u_{i}}\right]\right. \\
& \left.+n t\left[\frac{\sum_{x_{i} \in X_{2}} u_{i}\left\{\left(x_{A}^{r} x_{C}^{1-r}+\left(1-x_{A}\right)^{r}\left(1-x_{C}\right)^{1-r}\right)^{\frac{1}{r}}\right\}}{\sum_{x_{i} \in X_{2}} u_{i}}-\frac{\sum_{x_{i} \in X_{2}} u_{i}\left\{\left(x_{A}^{r}+\left(1-x_{A}\right)^{r}\right)^{\frac{1}{r}}\right\}}{\sum_{x_{i} \in X_{2}} u_{i}}\right]\right\} \\
& +\left\{n t\left[\frac{\sum_{x_{i} \in \mathrm{X}_{1}} u_{i}\left\{\left(x_{A}^{r} x_{C}^{1-r}+\left(1-x_{A}\right)^{r}\left(1-x_{C}\right)^{1-r}\right)^{\frac{1}{r}}\right\}}{\sum_{x_{i} \in X_{i}} u_{i}}-\frac{\sum_{x_{i} \in X_{i}} u_{i}\left\{\left(x_{A}^{r}+\left(1-x_{A}\right)^{r}\right)^{\frac{1}{r}}\right\}}{\sum_{x_{i} \in X_{i}} u_{i}}\right]\right.
\end{aligned}
$$




$$
\begin{gathered}
\left.+n t\left[\frac{\sum_{x_{i} \in \mathrm{X}_{2}} u_{i}\left\{\left(x_{A}^{r} x_{B}^{1-r}+\left(1-x_{A}\right)^{r}\left(1-x_{B}\right)^{1-r}\right)^{\frac{1}{r}}\right\}}{\sum_{x_{i} \in \mathrm{X}_{2}} u_{i}}-\frac{\sum_{x_{i} \in \mathrm{X}_{2}} u_{i}\left\{\left(x_{A}^{r}+\left(1-x_{A}\right)^{r}\right)^{\frac{1}{r}}\right\}}{\sum_{x_{i} \in \mathrm{X}_{2}} u_{i}}\right]\right\} \\
=n t\left[\frac{\sum_{i=1}^{n} u_{i}\left\{\left(x_{A}^{r} x_{B}^{1-r}+\left(1-x_{A}\right)^{r}\left(1-x_{B}\right)^{1-r}\right)^{\frac{1}{r}}\right\}}{\sum_{i=1}^{n} u_{i}}-\frac{\sum_{i=1}^{n} u_{i}\left\{\left(x_{A}^{r}+\left(1-x_{A}\right)^{r}\right)^{\frac{1}{r}}\right\}}{\sum_{i=1}^{n} u_{i}}\right] \\
+n t\left[\frac{\sum_{i=1}^{n} u_{i}\left\{\left(x_{A}^{r} x_{C}^{1-r}+\left(1-x_{A}\right)^{r}\left(1-x_{C}\right)^{1-r}\right)^{\frac{1}{r}}\right\}}{\sum_{i=1}^{n} u_{i}}-\frac{\sum_{i=1}^{n} u_{i}\left\{\left(x_{A}^{r}+\left(1-x_{A}\right)^{r}\right)^{\frac{1}{r}}\right\}}{\sum_{i=1}^{n} u_{i}}\right] . \\
=I_{R}^{\alpha, \beta}(A ; B ; U)+I_{R}^{\alpha, \beta}(A ; C ; U) .
\end{gathered}
$$

Hence, the result follows.

\subsection{Generalized 'Useful' R-Norm Fuzzy Information Improvement Measure}

Suppose, a fuzzy set $B$ is used as an approximation of fuzzy set $A$. A revision is made and $B$ is replaced by a new fuzzy set $E$. The difference between the original directed divergence measure $D(A, B)$ and the revised directed divergence measure $D(A, E)$ or the reduction achieved in ambiguity by revising the original set $B$ by a new set $E$ is called fuzzy information improvement. It is written as

$$
D(A, B)-D(A, E) .
$$

Corresponding to 'useful' R-norm fuzzy DDM defined in (10), we propose the 'useful' R-norm fuzzy information improvement measure as

$$
\begin{gathered}
D_{R}^{\alpha, \beta}(A, B ; U)-D_{R}^{\alpha, \beta}(A, E ; U)=\frac{R+\alpha-\beta}{\beta-R}\left[\frac{n \sum_{i=1}^{n} u_{i}}{\sum_{i=1}^{n} u_{i}}\left\{1-\left(\mu_{A}^{\frac{R+\alpha-\beta}{\alpha}}\left(x_{i}\right) \mu_{B}^{1-\frac{R+\alpha-\beta}{\alpha}}\left(x_{i}\right)+\left(\left(1-\mu_{A}\left(x_{i}\right)\right)^{\frac{R+\alpha-\beta}{\alpha}}\left(1-\mu_{B}\left(x_{i}\right)\right)^{1-\frac{R+\alpha-\beta}{\alpha}}\right)^{\frac{\alpha}{R+\alpha-\beta}}\right\}\right]\right. \\
-\frac{R+\alpha-\beta}{\beta-R}\left[\frac{n \sum_{i=1}^{n} u_{i}}{\sum_{i=1}^{n} u_{i}}\left\{1-\left(\mu_{A}^{\frac{R+\alpha-\beta}{\alpha}}\left(x_{i}\right) \mu_{E}^{1-\frac{R+\alpha-\beta}{\alpha}}\left(x_{i}\right)+\left(\left(1-\mu_{A}\left(x_{i}\right)\right)^{\frac{R+\alpha-\beta}{\alpha}}\left(1-\mu_{E}\left(x_{i}\right)\right)^{1-\frac{R+\alpha-\beta}{\alpha}}\right)^{\frac{\alpha}{R+\alpha-\beta}}\right\}\right] .\right.
\end{gathered}
$$

When $\alpha=1, \beta=1 \& u_{i}=1,(28)$ reduces to the measure given by Hooda and Bajaj [8].

\subsection{Monotone Behaviour of 'Useful' RTAM (14)}

We study the monotonic nature of the proposed measure in the given limits of $R, \alpha$ and $\beta$. We take two fuzzy sets $A \& B$ defined respectively as $A=(0.65,0.23,0.82,0.44,0.97,0.31)$ and $B=(0.42,0.28,0.05,0.90,0.73,0.61)$ with utility distribution $U=(5,2,4,1,3,6)$ and $n=6$.

Table 9. Values of Measure (14) for Fixed $\alpha$ and $\beta$

\begin{tabular}{|c|c|c|c|c|c|c|c|c|}
\hline$R$ & 3 & 7 & 20 & 47 & 62 & 100 & 120 & 140 \\
\hline$I_{R}^{0.96,0.20}(A ; B ; U)$ & 14.6288 & 18.2923 & 20.9998 & 22.0255 & 22.2208 & 22.4580 & 22.5235 & 22.5704 \\
\hline$I_{R}^{0.620 .81}(A ; B ; U)$ & 15.4946 & 19.3770 & 21.5787 & 22.3048 & 22.4372 & 22.5958 & 22.6391 & 22.6701 \\
\hline$I_{R}^{0.59,0.59}(A ; B ; U)$ & 16.1326 & 19.6114 & 21.6497 & 22.3331 & 22.4585 & 22.6088 & 22.6499 & 22.6793 \\
\hline
\end{tabular}




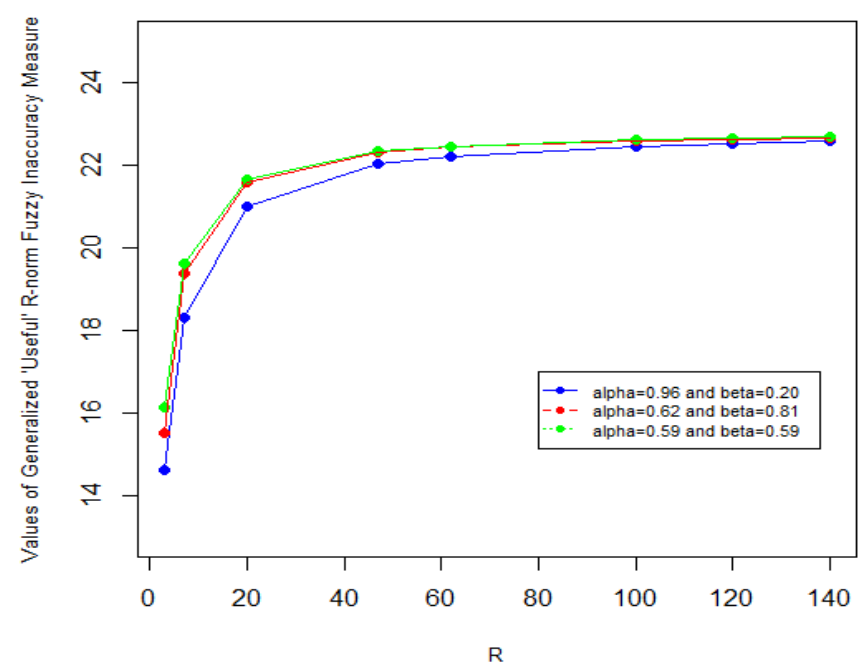

Fig.4. Graphical Overview of Measure (14) at Fixed Alpha and Beta

From Table 9, it becomes obvious that as we increase the value of $R$ and keep $\alpha \& \beta$ fixed; the 'useful' RTAM defined in (14) shows an increasing trend. We further observe that as $R$ increases the impact of parameters tend to vanish as the values of (14) coincide for higher values of $R$.

The increasing trend of measure (14) with respect to varying $R$ is depicted in the Fig. 4 by taking values of $\alpha \& \beta$ as $(0.96,0.20),(0.62,0.81) \&(0.59,0.59)$ respectively.

Table 10. Values of Measure (14) for Fixed $R$ and $\beta$

\begin{tabular}{|c|c|c|c|c|c|c|c|c|c|c|}
\hline$\alpha$ & 0.1 & 0.2 & 0.3 & 0.4 & 0.5 & 0.6 & 0.7 & 0.8 & 0.9 & 1.0 \\
\hline$I_{0.72}^{\alpha, 0.2}(A ; B ; U)$ & 12.9077 & 10.1040 & 8.8766 & 8.2124 & 7.8043 & 7.5315 & 7.3376 & 7.1933 & 7.0822 & 6.9941 \\
\hline$I_{5}^{\alpha, 0.02}(A ; B ; U)$ & 22.0423 & 21.2929 & 20.6018 & 19.9626 & 19.3702 & 18.8198 & 18.3074 & 17.8292 & 17.3820 & 16.9632 \\
\hline$I_{23}^{\alpha, 0.71}(A ; B ; U)$ & 22.6688 & 22.4843 & 22.3033 & 22.1257 & 21.9514 & 21.7802 & 21.6122 & 21.4473 & 21.2853 & 21.1261 \\
\hline
\end{tabular}

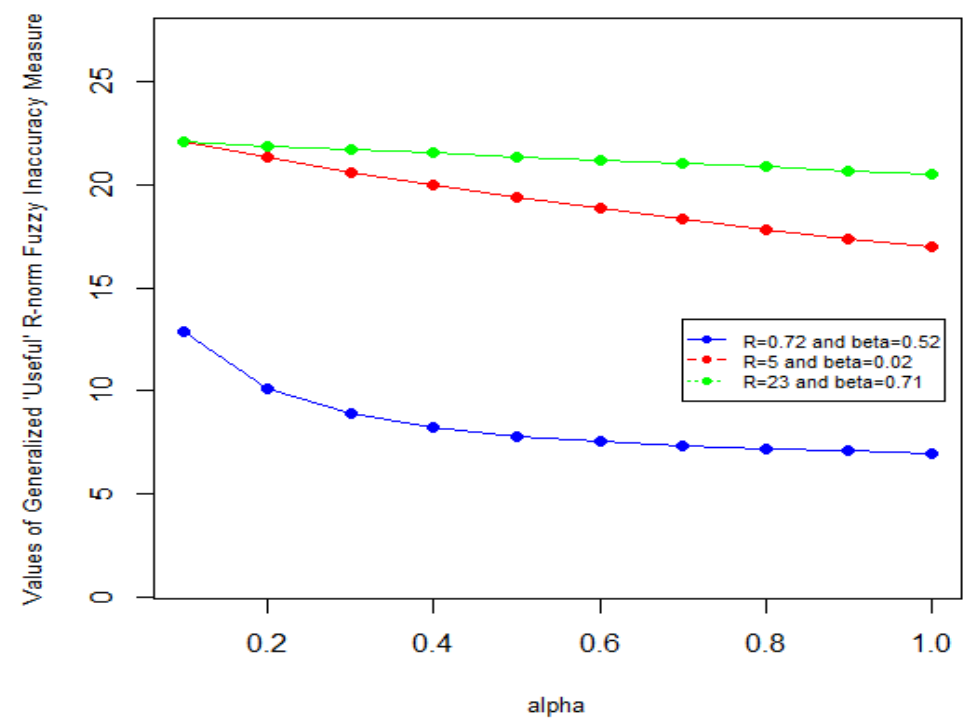

Fig.5. Graphical Overview of Measure (14) at Fixed $R$ and Beta

From Table 10, we can easily state that as the value of $\alpha$ increases ( $R \& \beta$ are fixed), the value of measure (14) decreases. Although, the value of measure (14) changes if we alter the values of $R \& \beta$ but the trend (that is decreasing) remains the same. Thus, there is a negative relation between $\alpha$ and the measure (14). This relation is presented graphically in the Fig. 5 by taking values of $R \& \beta$ as $(0.72,0.52),(5,0.02) \&(23,0.71)$ respectively. 
Table 11. Values of Measure (14) for Fixed $\alpha$ and $R$

\begin{tabular}{|c|c|c|c|c|c|c|c|c|c|c|}
\hline$\beta$ & 0.1 & 0.2 & 0.3 & 0.4 & 0.5 & 0.6 & 0.7 & 0.8 & 0.9 & 0.93 \\
\hline$I_{2}^{0.98, \beta}(A ; B ; U)$ & 12.7685 & 12.5284 & 12.2777 & 12.0157 & 11.7418 & 11.4555 & 11.1561 & 10.8431 & 10.5159 & 10.4149 \\
\hline$I_{0.94}^{0.16, \beta}(A ; B ; U)$ & 17.1752 & 16.6593 & 16.0419 & 15.2907 & 14.3596 & 13.1811 & 11.6573 & 9.6616 & 7.1933 & 6.4750 \\
\hline$I_{23}^{0.63, \beta}(A ; B ; U)$ & 21.7579 & 21.7533 & 21.7487 & 21.7441 & 21.7394 & 21.7347 & 21.7299 & 21.7252 & 21.7204 & 21.7189 \\
\hline
\end{tabular}

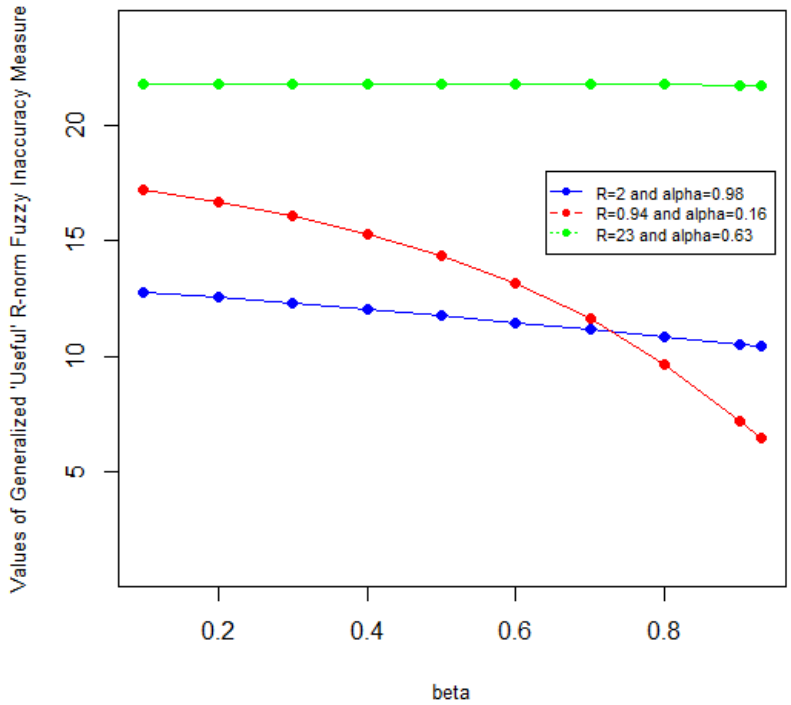

Fig.6. Graphical Overview of Measure (14) at Fixed R and Alpha

From Table 11, we infer that the measure defined in (14) decreases as the value of $\beta$ increases keeping $R \& \alpha$ fixed. Thus, there is a negative relation between $\beta$ and the measure (14). This relation is shown in the Fig. 6 by taking values of $R \& \alpha$ as $(2,0.98),(0.94,0.16) \&(23,0.63)$ respectively.

From the Fig. 6, we further conclude that there is a minimal decrease in the value of (14) at $R=23 \& \alpha=0.63$. Also, (14) decreases sharply when we take $R<1$.

Since $R>\beta$, we have taken the value of $\beta$ upto 0.93 only.

\subsection{Application of 'Useful' R-Norm Total Ambiguity Measure}

In this section, we demonstrate the application of the proposed 'useful' RTAM (14) in the context of multi-criteria decision making. Decision making basically concerns with making best choice from all the available choices. There are many situations where the decision makers find it hard to make the best choice since the information available is very little or vague about the alternatives. So, the decision makers present their preferences in the form of fuzzy information. Various fuzzy MCDM approaches have been established and are employed to a variety of fields.

Suppose $D=\left\{D_{1}, D_{2}, \ldots, D_{l}\right\}$ be a set choices and $K=\left\{K_{1}, K_{2}, \ldots, K_{m}\right\}$ be a set of criteria. Let $U=\left\{U_{1}, U_{2}, \ldots, U_{m}\right\}$ represent the respective importance of each criterion. The characteristics of the choice $D_{i}$ in terms of criteria $K_{j}$ are symbolized by the following fuzzy sets:

$$
D_{i}=\left\{\left\langle K_{j}, \zeta_{i j}\right\rangle ; K_{j} \in K\right\}, i=1,2, \ldots, l \& j=1,2, \ldots, m
$$

where $\zeta_{i j}$ represents the extent to which $D_{i}$ satisfies $K_{j}$.

The method for solving fuzzy MCDM problem in terms of the measure proposed in this paper is described in the steps given below by considering a numerical example.

Example: Suppose a person wants to admit his child in a school. He has to choose among the six options i.e., $D=\left\{D_{1}, D_{2}, \ldots, D_{6}\right\}$ and take a decision based on the six criteria: $1 . K_{1}$ : fee structure 2. $K_{2}:$ quality education $3 . K_{3}:$ status of school 4. $K_{4}$ : infrastructure 5. $K_{5}$ :distance from home to school $6 . K_{6}$ : co-curriculum activities. Let $U=(4,3,6,1,2,5)$ be the utility distribution with $n=6$. The six possible choices under the six criteria are to be evaluated by the decision maker in the following form: 
$D_{1}=\left\{\left\langle K_{1}, 0.82\right\rangle,\left\langle K_{2}, 0.95\right\rangle,\left\langle K_{3}, 0.76\right\rangle,\left\langle K_{4}, 0.79\right\rangle,\left\langle K_{5}, 0.88\right\rangle,\left\langle K_{6}, 0.41\right\rangle\right\} D_{2}=\left\{\left\langle K_{1}, 0.49\right\rangle,\left\langle K_{2}, 0.51\right\rangle,\left\langle K_{3}, 0.62\right\rangle,\left\langle K_{4}, 0.77\right\rangle,\left\langle K_{5}, 0.92\right\rangle,\left\langle K_{6}, 0.83\right\rangle\right\}$

$D_{3}=\left\{\left\langle K_{1}, 0.71\right\rangle,\left\langle K_{2}, 0.66\right\rangle,\left\langle K_{3}, 0.84\right\rangle,\left\langle K_{4}, 0.56\right\rangle,\left\langle K_{5}, 0.79\right\rangle,\left\langle K_{6}, 0.46\right\rangle\right\} D_{4}=\left\{\left\langle K_{1}, 0.65\right\rangle,\left\langle K_{2}, 0.89\right\rangle,\left\langle K_{3}, 0.55\right\rangle,\left\langle K_{4}, 0.67\right\rangle,\left\langle K_{5}, 0.81\right\rangle,\left\langle K_{6}, 0.59\right\rangle\right\}$

$D_{5}=\left\{\left\langle K_{1}, 0.87\right\rangle,\left\langle K_{2}, 0.74\right\rangle,\left\langle K_{3}, 0.71\right\rangle,\left\langle K_{4}, 0.54\right\rangle,\left\langle K_{5}, 0.69\right\rangle,\left\langle K_{6}, 0.62\right\rangle\right\} D_{6}=\left\{\left\langle K_{1}, 0.78\right\rangle,\left\langle K_{2}, 0.69\right\rangle,\left\langle K_{3}, 0.47\right\rangle,\left\langle K_{4}, 0.61\right\rangle,\left\langle K_{5}, 0.73\right\rangle,\left\langle K_{6}, 0.65\right\rangle\right\}$

Step 1: Obtain the positive-ideal solution $D^{+}$and negative-ideal solution $D^{-}$as

$$
D^{+}=\left\{\left\langle\xi_{1+}\right\rangle,\left\langle\xi_{2+}\right\rangle, \ldots,\left\langle\xi_{m+}\right\rangle\right\} \& D^{-}=\left\{\left\langle\xi_{1-}\right\rangle,\left\langle\xi_{2-}\right\rangle, \ldots,\left\langle\xi_{m-}\right\rangle\right\} .
$$

where for each $j=1,2, \ldots, m$

$$
\left\langle\xi_{j+}\right\rangle=\left\langle\max _{i} \xi_{i j}\right\rangle \&\left\langle\xi_{j-}\right\rangle=\left\langle\min _{i} \xi_{i j}\right\rangle .
$$

Thus, $D^{+}$and $D^{-}$are obtained respectively as:

$$
D^{+}=\left\{\left\langle K_{1}, 0.87\right\rangle,\left\langle K_{2}, 0.95\right\rangle,\left\langle K_{3}, 0.84\right\rangle,\left\langle K_{4}, 0.79\right\rangle,\left\langle K_{5}, 0.92\right\rangle,\left\langle K_{6}, 0.83\right\rangle\right\} D^{-}=\left\{\left\langle K_{1}, 0.49\right\rangle,\left\langle K_{2}, 0.51\right\rangle,\left\langle K_{3}, 0.47\right\rangle,\left\langle K_{4}, 0.54\right\rangle,\left\langle K_{5}, 0.69\right\rangle,\left\langle K_{6}, 0.41\right\rangle\right\}
$$

Step 2: Values of $I_{R}^{\alpha, \beta}\left(D^{+} ; D_{i} ; U\right)$ and $I_{R}^{\alpha, \beta}\left(D^{-} ; D_{i} ; U\right)$ where $i=1,2, \ldots, 6$ are obtained respectively in the following tables as per the expression (14).

Table 12. Values of $I_{R}^{\alpha, \beta}\left(D^{+} ; D_{1} ; U\right)$

\begin{tabular}{|c|c|c|}
\hline & $\alpha=0.26$ & $\alpha=0.72$ \\
& $\beta=0.37$ & $\beta=0.81$ \\
& $R=0.97$ & $R=32$ \\
\hline$I_{R}^{\alpha, \beta}\left(D^{+} ; D_{1} ; U\right)$ & 2.4072 & 2.5345 \\
\hline$I_{R}^{\alpha, \beta}\left(D^{+} ; D_{2} ; U\right)$ & 2.9416 & 3.0413 \\
\hline$I_{R}^{\alpha, \beta}\left(D^{+} ; D_{3} ; U\right)$ & 2.6971 & 2.7961 \\
\hline$I_{R}^{\alpha, \beta}\left(D^{+} ; D_{4} ; U\right)$ & 2.6722 & 2.8549 \\
\hline$I_{R}^{\alpha, \beta}\left(D^{+} ; D_{5} ; U\right)$ & 2.1455 & 2.1713 \\
\hline$I_{R}^{\alpha, \beta}\left(D^{;} ; D_{6} ; U\right)$ & 2.9943 & 3.2245 \\
\hline
\end{tabular}

Table 13. Values of $I_{R}^{\alpha, \beta}\left(D^{-} ; D_{i} ; U\right)$

\begin{tabular}{|c|c|c|}
\hline & $\alpha=0.26$ & $\alpha=0.72$ \\
& $\beta=0.37$ & $\beta=0.81$ \\
& $R=0.97$ & $R=32$ \\
\hline$I_{R}^{\alpha, \beta}\left(D^{-} ; D_{1} ; U\right)$ & 9.5807 & 15.0886 \\
\hline$I_{R}^{\alpha, \beta}\left(D^{\circ} ; D_{2} ; U\right)$ & 6.3635 & 8.6181 \\
\hline$I_{R}^{\alpha, \beta}\left(D^{\circ} ; D_{3} ; U\right)$ & 6.0413 & 8.1151 \\
\hline$I_{R}^{\alpha, \beta}\left(D^{\circ} ; D_{4} ; U\right)$ & 5.4506 & 7.3862 \\
\hline$I_{R}^{\alpha, \beta}\left(D^{\circ} ; D_{5} ; U\right)$ & 6.4722 & 8.7976 \\
\hline$I_{R}^{\alpha, \beta}\left(D^{\circ} ; D_{6} ; U\right)$ & 4.7101 & 5.7181 \\
\hline
\end{tabular}

Step 3: Value of relative 'useful' RTAM $I_{R}^{\alpha, \beta}\left(D_{i} ; U\right)$ of each $D_{i}$ with respect to $D^{+}$and $D^{-}$are computed as per the below formula

$$
I_{R}^{\alpha, \beta}\left(D_{i} ; U\right)=\frac{I_{R}^{\alpha, \beta}\left(D^{+} ; D_{i} ; U\right)}{I_{R}^{\alpha, \beta}\left(D^{+} ; D_{i} ; U\right)+I_{R}^{\alpha, \beta}\left(D^{-} ; D_{i} ; U\right)}, \forall i
$$

The results are calculated for $\alpha=0.26,0.72 ; \beta=0.37,0.81 \& R=0.97,32$ in the subsequent table: 
Table 14. Values of $I_{R}^{\alpha, \beta}\left(D_{i} ; U\right)$

\begin{tabular}{|c|c|c|}
\hline & $\alpha=0.26$ & $\alpha=0.72$ \\
$\beta=0.37$ & $\beta=0.81$ \\
& $R=0.97$ & $R=32$ \\
\hline$I_{R}^{\alpha, \beta}\left(D_{1} ; U\right)$ & $\mathbf{0 . 2 0 0 8}$ & $\mathbf{0 . 1 4 3 8}$ \\
\hline$I_{R}^{\alpha, \beta}\left(D_{2} ; U\right)$ & 0.3161 & 0.2608 \\
\hline$I_{R}^{\alpha, \beta}\left(D_{3} ; U\right)$ & 0.3086 & 0.2562 \\
\hline$I_{R}^{\alpha, \beta}\left(D_{4} ; U\right)$ & 0.3289 & 0.2787 \\
\hline$I_{R}^{\alpha, \beta}\left(D_{5} ; U\right)$ & 0.2489 & 0.1979 \\
\hline$I_{R}^{\alpha, \beta}\left(D_{6} ; U\right)$ & 0.3886 & 0.3605 \\
\hline
\end{tabular}

From the above Table 14, we get the following ranking order of available choices

$$
D_{1} \succ D_{5} \succ D_{3} \succ D_{2} \succ D_{4} \succ D_{6}
$$

This implies $D_{1}$ is the most appropriate choice.

\section{Conclusion}

In this manuscript, we have presented new generalized measure of 'useful' R-norm inaccuracy and 'useful' Rnorm total ambiguity. The fundamental properties of both the proposed measures are stated which validate these measures. The particular cases are also discussed for both the measures. Further, the information improvement measures are studied. The monotonic property of both the inaccuracy measures is discussed with respect to the parameters introduced. In the end, the application to multi-criteria decision making of 'useful' R-norm total ambiguity measure is presented.

\section{References}

[1] D. Kerridge, "Inaccuracy and inference", Journal of Royal Statistical Society B, vol. 23, no. 1, 184-194, 1961.

[2] C. E. Shannon, "A mathematical theory of communication", Bell System Technical Journal, vol. 27, 379-423, 623-656, 1948.

[3] D. S. Hooda and A. Ram, "Characterization of a generalized measure of R-norm entropy", Caribbean Journal of Mathematical Computing Science, vol. 8(1 \& 2), 18-31, 1998.

[4] L. A. Zadeh, "Fuzzy sets", Information and Control, vol. 8, No. 3, 338-353, 1965.

[5] R. K. Verma and B. D. Sharma, "A measure of inaccuracy between two fuzzy sets", Cybernetics and Information Technologies, vol. 11, no. 2, 13-23, 2011.

[6] D. S. Hooda and D. K. Sharma, "Generalized R-norm information measures", Journal of Applied Mathematics, Statistics and Informatics, vol. 4, no.2, pp.153-168, 2008.

[7] D. E. Boekee and V. D. Lubbe, "The R-norm information measure", Information and Control, 45,136-155, 1980.

[8] D. S. Hooda and R. K. Bajaj, "On generalized R-norm measures of fuzzy information”, Journal Of Applied Mathematics, Statistics And Informatics, vol. 4, no. 2, 199-212, 2008.

[9] R. Verma and B. D. Sharma, "A new inaccuracy measure for fuzzy sets and its application in multi-criteria decision making", International Journal of Intelligent Systems and Applications, vol. 05, 62-69, 2014.

[10] A. H. Bhat, M. A. Bhat, M. A. K. Baig and S. Manzoor, "Noiseless coding theorems of generalized useful fuzzy inaccuracy measure of order alpha and type beta," International Journal of Fuzzy Mathematical Archive, vol. 13, no. 2, 135-143, 2017.

[11] A. H. Bhat, M. J. Dar and M. A. K. Baig, "Two parametric new generalized average code-word length and its bounds in terms of new generalized inaccuracy measure and their characterization”, Pakistan Journal of Statistics, vol. 34, no. 2, 147-162, 2018.

[12] S. M. Sofi, S. Peerzada and A. H. Bhat, "Two parametric generalized 'useful' R-norm information measure \& its coding theorems", Pakistan Journal of Statistics, vol. 35, no. 2, 109-126, 2019.

[13] S. M. Sofi, S. Peerzada, R. Jamil and M. A. K. Baig, “Applications of Two Parametric Generalized 'Useful' R-Norm Directed Divergence Measures" unpublished.

[14] H. Theil, "Economics and information theory", North-Holland Publishing Company, Amesterdam, 1967.

[15] S. Peerzada, S. M. Sofi and R. Jamil, "On parametric generalized R-norm: inaccuracy and fuzzy inaccuracy information measures", Journal of Applied Probability and Statistics, vol. 15, no. 3, 79-92, 2020.

[16] S. M. Sofi, S. Peerzada and M. A. K. Baig, "Parametric generalizations of 'useful' R-norm fuzzy information measures", International Journal of Scientific Research in Mathematical and Statistical Sciences, vol. 5, no. 6, 164-169, 2018. 


\section{Authors' Profiles}

Saima Manzoor and Safina Peerzada were born in Srinagar, Jammu and Kashmir, India. They received the B.A. from Government College for Women, M.A. Road, Srinagar in 2010 and received Masters and M.Phil. degrees in Statistics from University of Kashmir, India in 2013 and 2017 respectively.

They have worked as teaching assistant in colleges of Kashmir in 2015. Since July 2017, they are pursuing Ph. D. in department of Statistics, University of Kashmir, Srinagar (India). Their research interests include information theory, coding theory, divergence measures, 'useful' information measures, fuzzy information measures and R-norm information measures.

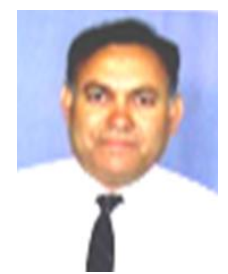

Mirza A. K. Baig was born in Bihar, India. He received the B.Sc. degree in Statistics Honors from Aligarh Muslim University, India in 1983. He has received Masters, M.Phil. and Ph.D. in Statistics from Aligarh Muslim University, India in the years 1985, 1987 and 1995 respectively. His major field of study is Information Theory and Applied Probability.

He has a work experience of 28 years. He was a gold medalist both in graduation and post-graduation. He has guided $7 \mathrm{Ph}$. D. and 12 M.Phil. scholars till date. He has served as head of the Department of Statistics, University of Kashmir, India, 190006. His research interests include information theory, fuzzy measures, information reliability. He has published more than 70 research papers till date. Presently, he is working as Head \& Professor in the Department of Statistics, University of Kashmir, India, 190006.

How to cite this paper: Saima Manzoor, Safina Peerzada, Mirza A. K. Baig, "Application of Generalized Measure of 'Useful' Rnorm Inaccuracy and Total Ambiguity", International Journal of Intelligent Systems and Applications(IJISA), Vol.13, No.1, pp.17-33, 2021. DOI: $10.5815 /$ ijisa.2021.01.02 